dass derselbe möglicherweise durch die Beobachtungen nicht zu constatiren ist. Das über die tägliche und jährliche Schwankung bereits Gesagte gilt unverändert auch für diesen Fall, da $\mu$ dasselbe ist.

Eine Bestätigung dieser vielleicht etwas gewagten Hypothese liegt in den neuerlich gemachten Erfahrungen, dass das elektrische Potential der Luft mit der Höhe linear zunimmt und einer täglichen und jährlichen Periode unterworfen ist, was sich aus dem Werthe des elektrischen Potentials unter den hier gemachten Annahmen:

leicht ergiebt.

$$
V=K_{1} \frac{e}{p}+\frac{e}{p^{2}} r \cos z\left(K_{1}+K \frac{h}{a}\right)
$$

Eine von der 'Talcott'schen Methode wesentlich verschiedene, ebenfalls sehr genaue Methode, welche an den

$$
\zeta-\zeta_{0}=(\nu-1)\left(\frac{a}{s}\right)^{2} \frac{e}{M}\left[K_{1}-1 /{ }_{4} K\left(\frac{a_{1}^{2}}{a^{2}}-x\right)\right] \sec \zeta \cos \delta \sin t
$$

Es wird demnach auch hier eine tägliche und eine jährliche Periode, letztere jedoch mit viel kleinerer Amplitude, auftreten müssen, wobei auch die Zeiten der Maxima und Minima gegen die aus Meridianbeobachtungen abgeleiteten verschieden sein müssen; da aber die Aenderungen der Zenithdistanzen im ersten Vertical in unseren Breiten etwa den zwölffachen Betrag der Polhöhenänderungen betragen, so würden, falls die aus Meridianbeobachtungen ge-

Wien-Ottakring 1891 Januar. mit hierzu geeigneten Instrumenten versehenen Sternwarten jedenfalls nicht unterlassen werden sollte, sind Beobachtungen im I. Vertical, und zwar ebenso Durchgangsbeobachtungen als Zenithdistanzmessungen; nur müsste die jetzt ubbliche Methode der Beobachtung der Durchgänge desselben Sterns im Ost- und West-Vertical verlassen werden, und statt dessen müssten ebenfalls Sternpaare in kleinen Zwischenräumen im Ost- und West.Verticale in sehr nahe gleichen Zenithdistanzen genommen werden. Azimuthänderungen, welche eine vorwiegende Fehlerquelle bei der bisherigen Methode sind, bleiben hier belanglos, können aber auch durch wiederholte Beobachtungen von Controllsternen in anderen Zenithdistanzen ermittelt werden.

Die Aenderung der Zenithdistanz im ersten Vertical in der Nähe des Zeniths in Folge der elektrischen Influenz wird;

folgerten Polhöhenänderungen wirklichen Lagenänderungen der Erdaxe entsprechen, die Aenderungen der Zenithdistanzen im ersten Vertical etwa $12 \mathrm{mal}$ so gross sein müssen. Es wäre daher wünschenswerth, wenn nebst den Polhöhenbestimnungen nach Talcott's Methode in möglichst verschiedenen Längen und Breiten auch Beobachtungen im ersten Vertical nach der hier berührten Methode vorgenommen würden.

N. Herz.

\title{
Bemerkung zu einer Aufgabe der Integralrechnung.
}

In der Mécanique céleste (Tome I, p. 40) von Herrn Tisserand findet man das Problem, eine Function $w \mathrm{zu}$ be. stimmen, welche den Bedingungen genügt:

(r) $\frac{\partial^{3} w}{\partial x^{3}}=0, \frac{\partial^{3} w}{\partial x^{2} \partial y}=0, \frac{\partial^{3} w}{\partial x \partial y^{2}}=0, \frac{\partial^{3} w}{\partial z^{3}}=0$ (2)

$\left\{\begin{array}{l}2 w\left(x \frac{\partial^{2} w}{\partial x^{2}}+y \frac{\partial^{2} w}{\partial x \partial y}\right)-\frac{\partial w}{\partial x}\left(x \frac{\partial w}{\partial x}+y \frac{\partial w}{\partial y}\right)=0 \\ 2 w\left(y \frac{\partial^{2} w}{\partial y^{2}}+x \frac{\partial^{2} w}{\partial x \partial y}\right)-\frac{\partial w}{\partial y}\left(x \frac{\partial w}{\partial x}+y \frac{\partial w}{\partial y}\right)=0\end{array}\right.$

Diese höchst einfache Aufgabe kann man noch in einer etwas anderen Form als a. a. O. lösen, wenn man für $w$ eine quadratische Form von $x, y, z$ setzt:

$w=a x^{2}+2 b x y+c y^{2}+2 f x z+2 g y z+h z^{2}$.

Dann verwandeln sich die Gleichungen (2) in

$$
\begin{aligned}
& z\left(\frac{\partial w}{\partial x} \frac{\partial w}{\partial z}-\frac{\partial^{2} w}{\partial x \partial z} 2 w\right)=0 \\
& z\left(\frac{\partial w}{\partial y} \frac{\partial w}{\partial z}-\frac{\partial^{2} w}{\partial y \partial z} z w\right)=0 .
\end{aligned}
$$

Hieraus folgt entweder $z=0$ und damit

$$
w=a x^{2}+2 b x y+c y^{2} \text {, }
$$

oder

$$
\begin{aligned}
& \frac{\partial w}{\partial x} \frac{\partial w}{\partial z}-\frac{\partial^{2} w}{\partial x \partial z} \mathbf{2 w}=0 \\
& \frac{\partial w}{\partial y} \frac{\partial w}{\partial z}-\frac{\partial^{2} w}{\partial y \partial z} 2 w=0 .
\end{aligned}
$$

Dann muss aber auch sein

$$
\left(\frac{\partial w}{\partial z}\right)^{2}-\frac{\partial^{2} w}{\partial z^{2}} 2 w=。
$$

wie man gleich findet, wenn man die drei letzten Gleichungen mit $x, y, z$ multiplicirt und addirt. Aus der letzten Gleichung folgt aber

$$
\begin{array}{ll}
w=\left(\frac{\mathrm{I}}{2} \frac{\partial w}{\partial z}\right)^{2}: \frac{\mathrm{I}}{2} \frac{\partial^{2} w}{\partial z^{2}} ; & \text { d. h. für } z=\mathbf{r} \\
w=\frac{(f x+g y+h)^{2}}{h}, & {[h]>0 .}
\end{array}
$$

Ueberhaupt folgt aus dem Euler'schen Satze über homogene Functionen, dass, wenn $f\left(x_{1}, x_{2}, \ldots x_{n}\right)$ eine quadratische Form von $n$ Variabeln vorstellt,

$$
f=a_{11} x_{1}^{2}+2 a_{12} x_{1} x_{2}+\cdots+a_{n n} x_{n}^{2},
$$

und die Gleichungen stattfinden

$$
\frac{\partial f}{\partial x_{i}} \frac{\partial f}{\partial x_{n}}-\frac{\partial^{2} f}{\partial x_{i} \partial x_{n}} 2 f=0,(i=1,2, \ldots n-1)
$$

die Form $f=\frac{1}{a_{i i}}\left(a_{i 1} x+a_{i 2} x_{2}+\cdots+a_{i n} x_{n}\right)^{i}$ ist, wo $a_{i k}=a_{k i}$ und $a_{i i}$ als von $\circ$ verschieden vorausgesetzt wird.
L. de Ball. 